\title{
Problematización, Historia del Pensamiento y Arqueología en Michel Foucault ${ }^{*}$ \\ Problematization, History of Thought and Archeology in Michel Foucault
}

\author{
Por: Guillermo Vega * \\ Facultad de Humanidades \\ Universidad Nacional del Nordeste \\ Resistencia. Chaco. Argentina \\ Email: guivega1978@gmail.com
}

Fecha de recepción: 13/09/2019

Fecha de aprobación: 7/10/2019

Doi: http://dx.doi.org/10.30972/nvt.0154011

\section{Resumen}

El presente escrito sostiene que es posible recuperar como legado foucaulteano -para los debates contemporáneos acerca de la especificidad del trabajo filosófico- la centralidad de la noción de problematización, comprendida como indicador de un objeto específico de análisis; la arqueología, como modo privilegiado de acceso al mismo; y la historia del pensamiento como empresa mayor en la cual objeto y modo de acceso se inscriben. Para ello, el escrito indaga, en una primera parte, la especificidad de la problematización foucaulteana como objeto de interés para el trabajo intelectual. En esta instancia se discuten dos sentidos de la noción de problematización a través de algunas referencias realizadas sobre el trabajo de Carol

\footnotetext{
* La temática de este artículo forma parte de uno de los objetos de indagación que acomuna a investigadores y becarios en el Proyecto de Investigación 16W002 de la Secretaría General de Ciencia y Técnica de la Universidad Nacional del Nordeste.

* Doctor en Filosofía por la Universidad Nacional del Nordeste. 2017. Magister en Ciencias Sociales y Humanidades con orientación en Filosofía Social y Política por la Universidad Nacional de Quilmes. 2014. Profesor y Licenciado en Filosofía por la Universidad Nacional del Nordeste (UNNE). Profesor adjunto, con dedicación exclusiva, en la cátedra de "Filosofía del Derecho" en la Facultad de Humanidades de la UNNE. 2004 hasta la actualidad. Profesor adjunto en la cátedra "Filosofía Política", con dedicación simple, en la Facultad de Humanidades de la UNNE. 2017 hasta la actualidad. Docente investigador con categoría III de acuerdo con el Programa Nacional de Incentivos del Ministerio de Educación de la Nación. Director del Proyecto de Investigación PI 16W002, radicado en la UNNE, sobre estudios en gubernamentalidad. Integrante del Proyecto Tipo A, № 2366, radicado en la Universidad Nacional de Salta (UNSa), sobre gubernamentalidades neoliberales. Coordinador del Área de Posgrado de la Facultad de Humanidades de la UNNE. Director del Dpto. de Filosofía de la Facultad de Humanidades de la UNNE.
} 
Bacchi. En un segundo momento, se explora el modo en que el análisis de las formas de problematización se integra en el proyecto de una historia del pensamiento. Para ello se vinculan las nociones de pensamiento y problematización a partir de la especificidad de los juegos de verdad. Esta dirección indica el camino a la última parte del escrito, en la que se recuperan los elementos constitutivos de la arqueología, asumida como modo de acceso privilegiado para el análisis de las formas de problematización, en el marco mayor de una historia del pensamiento.

\title{
Palabras clave
}

Historia de los sistemas de pensamiento; formas de problematización; juegos de verdad; arqueología.

\begin{abstract}
This paper maintains that it is possible to recover as a Foucauldain legacy - for contemporary debates about the specificity of philosophical work - the centrality of the notion of problematization, understood as an indicator of a specific object of analysis; archeology, as a privileged way of accessing it; and the history of thought as a major enterprise in which object and mode of access are registered. Thus, the paper investigates, in the first part, the specificity of Foucauldian problematization as an object of interest for intellectual work. In this instance, two senses of the notion of problematization are discussed through some references made on Carol Bacchi's work. In a second moment, the paper explore the way in which the analysis of the forms of problematization is integrated into the project of a history of thought. For this, the notions of thought and problematization are linked from the specificity of the games of truth. This direction indicates the path to the last part of the writing, in which the constituent elements of archeology are recovered - assumed as a privileged access mode for the analysis of the problematization forms - in the greater framework of a history of thought.
\end{abstract}




\section{Guillermo Vega}

\section{Keywords}

History of thought systems; forms of problematization; games or truth; archeology.

\section{1.- Las "formas de problematización" como objeto de indagación}

Hacia comienzos de 1980, las intervenciones públicas de Foucault y las ediciones de los volúmenes II y III de Historia de la sexualidad dejaron en claro que las preocupaciones del filósofo francés se habían desplazado desde la temática concerniente al poder, ampliamente desarrollada a lo largo de toda la década del 70, hacia un nuevo objeto de indagación: las prácticas -individuales o colectivas- de constitución de subjetividad. Estas últimas investigaciones se desarrollaron de forma paralela a la inquietud por la especificidad del trabajo intelectual, expresada en varias entrevistas y conferencias concedidas a lo largo de los años comprendidos entre 1978 y 1984 (Foucault, 1999 y 2015). Por este período tienen lugar las reflexiones en torno de la crítica, la llustración y la ontología del presente, objetos que se volverán figuras centrales de una concepción de la labor filosófica que, para Foucault, tendrán su emergencia en Kant y se proyectarán luego en Nietzsche y Hegel, hasta definir su propia propuesta (Foucault, 2009: 39). No resulta arriesgado, entonces, afirmar que la especificidad o singularidad del pensamiento filosófico, los efectos políticos concretos que el mismo puede generar sobre los límites históricos de lo que somos y la relación compleja trazada entre el primer problema y el segundo constituyen un foco de preocupación intensa durante los últimos años de vida del filósofo francés. De aquí que no resulte casual el despliegue simultáneo de ambos tópicos (Vega, 2012).

Sin embargo, y más allá de los linajes filosóficos a los que Foucault adscribe su ascendencia o de la importancia que los términos Aufklärung y crítica adquieren por esos tiempos, el conjunto de las reflexiones cifradas sobre la temática de la especificad del trabajo intelectual parece encontrar un punto de condensación en la noción de "problematización". El sentido de dicho término se articula, tanto en las intervenciones foucaulteanas del período destacado como en el segundo tomo de la Historia de la 
sexualidad, en al menos dos órdenes semánticos: por un lado, problematización alude a un determinado tipo de abordaje, a un modo de vincularse con los objetos de conocimiento, a una manera de acceder a ellos; en otras palabras, problematización equivale a la actividad de problematizar, esto es, su sentido inmediato mienta un tipo de práctica, una forma de hacer algo, un procedimiento, una conducta. A partir de este primer significado se abre todo un campo de pensamiento que anuda la labor del intelectual -la problematización- a los procesos de transformación de sí, en fuerte consonancia con los desarrollos teóricos foucaulteanos del período llamado "ético". Esta apuesta descansa en un circuito de ida y vuelta que permite recorrer la distancia que separa el ámbito de la subjetividad de la práctica del pensamiento/problematización, tornando a una condición de la modificación de la otra y viceversa. ${ }^{1}$ En otras palabras, la constitución de una "estética de la existencia", tanto como punto de partida como de llegada de la actividad crítica del pensamiento y vinculada, asimismo, a efectos políticos en el plano de los límites identitarios histórica y socialmente construidos, resulta el corolario necesario de este primer sentido del término problematización. ${ }^{2}$ Por otro lado, por "problematización" Foucault parece comprender un dominio complejo caracterizado por la serie de acontecimientos que vuelve algo verdadero, es decir, las condiciones que permiten que sujetos, objetos y conceptos se construyan en el juego de lo verdadero y de lo falso y, en consecuencia, se vuelvan pasibles de ser pensados. Bajo este segundo significado, "problematización" alude a una "superficie" sobre la cual son posibles los elementos del pensamiento o, en otras palabras, bajo esta expresión se designa el a priori histórico que oficia de condición de posibilidad de todo pensamiento posible. Para expresarlo en términos didácticamente- cercanos a la filosofía kantiana: problematización es la "forma" del pensamiento; o bien, la forma que, puesta en relación con la materialidad de los

\footnotetext{
${ }^{1}$ Este tipo de preocupaciones pueden encontrarse en aquellas intervenciones que se centran en el modo de vida del intelectual, en las elecciones mínimas relativas a la forma de investigar, etc (Brossat y Chevallier, 2016).

${ }^{2}$ Una excelente compilación de textos foucaulteanos, organizados a partir de esta lectura, es la realizada por Jorge Yágüez y publicada por Biblioteca Nueva (Foucault, 2015). También se puede tomar como representativo de la primacía de la problematización como actividad el artículo de Díaz Marsá (2008).
} 


\section{Guillermo Vega}

acontecimientos históricos, hace posible la emergencia del pensamiento, ${ }^{3}$ en tanto conjunto de elementos y circuitos de recorridos posibles, encabalgado en un régimen de verdad. De aquí que Foucault sostenga que hacer una "Historia del Pensamiento" consiste en encontrar la "forma general de problematización" que ha vuelto posibles los pensamientos como series alternadas de inquietudes y respuestas.

La ambigüedad del término (Potte-Bonneville, 2007: 233), construida alrededor de los dos sentidos mencionados, ha sido recuperada por algunos pensadores que promovieron la distinción semántica como diferencia conceptual, y otros que ensayaron síntesis en las que las distancias se estrechan hasta fundir ambos sentidos. ${ }^{4}$ La importancia de estas lecturas -y sus apuestas- está depositada, fundamentalmente, en las expectativas que los cientistas sociales o los filósofos prácticos tienen en relación con el estatuto "metodológico" que intentan asignar al término problematización. ${ }^{5}$ Sin embargo, echar mano y disputar la efectividad o potencialidad metodológica de esta noción obliga a revisar la relevancia y especificidad de la arqueología y la genealogía, en tanto perspectivas singulares y complementarias en el modo de acceso a los objetos de estudio, para ponerlas en relación con la más joven noción de problematización.

\footnotetext{
3 "Hay una relación original entre el pensamiento y la realidad en el proceso de problematización" (Foucault, 2017: 282).

${ }^{4}$ Para ilustrar al primer grupo, basta con considerar las observaciones de C. Gordon, quien en la reseña de un diccionario terminológico foucaulteano sostiene, en relación con la palabra "problematización": "It seems to have been in 1983 that Foucault had the idea that problematization could be a useful term for defining his work, both present and past. He had this idea in more than one form. One form occurs in Foucault's later revision for French publication of material based on a discussion with Dreyfus and Rabinow in April 1983, where Foucault talks about problematization as an activity which characterizes his work, and the work of thought in general; another occurs in the introduction to The Use of Pleasures, in the concluding remarks of the autumn 1983 lectures on parresia in Berkeley, and in a 1984 interview with François Ewald, where Foucault talks about problematizations (in the plural) as historical objects of his investigations. Subject to correction, I cannot find a location where Foucault combines or connects these two usages" (Gordon, 2016: 95). Para exponer un ejemplo del segundo grupo, ver el estudio introductorio de Jorge Yágüez a la compilación de textos de Foucault ya referenciada (Foucault, 2015: 11-74).

${ }^{5}$ Para una discusión frente a las apuestas de las ciencias sociales y la construcción de un debate entre posiciones críticas y descriptivas a partir de la recuperación del entrecruzamiento entre Foucault y Dewey, ver Barnett (2015).
} 
La politóloga áustralocanadiense Carol Bacchi ${ }^{6}$ sugiere abordar la ambigüedad que parece habitar la noción de "problematización" distinguiendo entre un uso del término, en tanto verbo, y otro, como sustantivo (Bacchi, 2012: 1 y 2015: 2). Así, la problematización, como verbo, reconduciría a la actividad de problematizar, de transformar algo en un problema. Bacchi sugiere que bajo esta inflexión se ha reunido buena parte de las derivas críticas del pensamiento social. Su referencia a Paulo Freire contribuye a ilustrar el modo en que la actividad de problematizar se presenta, para la tradición crítica, como un modo de poner en cuestión las verdades aceptadas, o manipuladas ideológicamente (Bacchi, 2012: 1). La noción de problematización, asumida en este registro por algunos teóricos sociales, encontraría en la vieja idea de "crítica" la lógica de su sentido. Justo por ello, Bacchi indica la distancia que se traza entre esta manera de asumir el problematizar y el modo en que lo hace Foucault, dado que: "...problematization is more a description of thinking as a practice than a diagnosis of ideological manipulation" (Bacchi, 2012: 1).

Como sustantivo, la noción de problematización sugiere, en general, atender los resultados del proceso problematizador, ${ }^{7}$ esto es, la manera en que objetos, comportamientos, etc., se presentan articulados y definidos como problemas. La distinción así planteada -verbo y sustantivo- subsume las particulares del segundo uso al primero, puesto que la problematización como "sustantivo", esto es, como "resultado de un proceso", invita a pensar en el conjunto de acontecimientos o prácticas que han tornado algo en un problema. $^{8}$ En otras palabras, según el diagnóstico que $\mathrm{C}$. Bacchi realiza sobre los usos de la noción de problematización en ciencias sociales, el lugar de los procesos/comportamientos parece ser fundamental al momento de plantear una aproximación a los problemas y a las problematizaciones.

\footnotetext{
${ }^{6}$ Carol Bacchi ha desarrollado la perspectiva metodológica del WPR (What's the Problem Represented to be?) como modo de evaluación y análisis de las políticas públicas. En este sentido, su propuesta constituye un aporte interesante al desarrollo contemporáneo de las Ciencias Sociales y un desafío a posiciones constituidas alrededor de la temática de la problematización, tales como el Problem Solving. Para una presentación clara de su posición, ver Bacchi (2010).

7 Cfr., "As a noun, problematizations generally refer to the outcomes of the processes of problematizing..." (Bacchi, 2015: 3).

${ }^{8}$ Cfr., "...at times, the noun form appears simply as the nominalization of the process of problematizing" (Bacchi, 2015: 3).
} 
Esta ambivalencia, reportada por el cuadro de los usos contemporáneos del término, pareciera advertirse incluso en el modo en que Bacchi lee a Foucault: "Foucault employs the term "problematization" in two ways: first, to describe his method of analysis and, second, to refer to a historical process of producing objects for thought" (Bacchi, 2012: 1). Para la ampliación de la idea de "método de análisis", Bacchi retoma un pasaje de Roger Deacon, con quien afirma: “...the point of analysis is not to look for the one correct response to an issue but to examine how it is "questioned, analysed, classified and regulated" at "specific times and under specific circunstances" (Deacon, 2000: 127)" (Bacchi, 2012: 1). El segundo sentido foucaulteano se orienta sobre las condiciones (procesos históricos) que han proyectado sobre determinados objetos el estatuto de problemas, ${ }^{9}$ así como sobre el modo en que tales objetos han sido moldeados para hacerse pensables.

Una consecuencia concreta de este punto de vista resulta en la relevancia que adquiere la perspectiva genealógica sobre la arqueológica, a través de la búsqueda del origen y la procedencia -Herkunft y Entstehung- de los problemas (Foucault, 1997). El acento puesto en la problematización, comprendida como "conjunto de procesos históricos de constitución de problemas", corre en forma paralela a una restitución débil de los objetivos de la perspectiva crítica, en su sentido tradicional de denuncia. En efecto, Bacchi sostiene, casi en consonancia con la recuperación del espíritu crítico del pensamiento freireano: "The main purpose of studying problematization, therefore, is to "dismantle" objects (e.g. "sexuality", "madness") as taken-for-granted fixed essences... and to show how they have come to be" (Bacchi, 2012: 2). Esta lectura resuena en armonía con propuestas que vinculan problematización y eventualización, en tanto técnicas de pensamiento o estrategias metodológicas legadas por el filósofo francés. $^{10}$ En resumidas cuentas, luego de intentar caracterizar el objeto "problematización" (así como su empleo contemporáneo), Bacchi no deja lo suficientemente claro cuál es la forma singular en que su abordaje permitiría

\footnotetext{
${ }^{9}$ Es decir, han distribuido objetos bajo un cierto régimen de problematicidad.

${ }^{10} \mathrm{Cfr}$., “Ambas nociones (la de «eventualización» y problematización) permiten comprender la forma cómo Foucault operaba en la formulación de sus preguntas y, sobre todo, en algunos de los criterios de procedimiento que instrumentalizaba en sus trabajos" (Restrepo, 2008: 114).
} 
establecer una distinción específica con respecto a los modos más clásicos en que se han estructurado las perspectivas críticas de los procesos de reificación.

A una cierta distancia de esta lectura, el objetivo perseguido en este escrito es abordar el segundo sentido del término "problematización", esto es, en tanto objeto de indagación, e intentar restituir el valor que la arqueología tiene para un ejercicio analítico volcado sobre el mismo. La tesis barajada sugiere que al hacer las preguntas que realiza la analítica arqueológica se prefigura un objeto en cierta medida diferente de aquel que responde por las inquisiciones genealógicas. De todas maneras, no se trata en medida alguna de tener que optar entre el trabajo del arqueólogo y el del genealogista, sino de intensificar la mirada arqueológica para que, a partir de la misma, se haga visible la problematización como un objeto que responde tanto a los procesos que han convertido algo en un problema (y aquí, ciertamente, la mirada genealógica no deja de ser fundamental), como a las prácticas de verdad que, en el plano del discurso, compelen a que ciertos elementos discursivos adquieran el estatuto de objetos del pensamiento $y$, por lo tanto, pasen a constituir el orden de la experiencia posible.

\section{2.- La "historia del pensamiento" como empresa filosófica}

Uno de los textos foucaulteanos fundamentales con respecto a la idea de problematización es la "Introducción" al volumen segundo de la Historia de la sexualidad. Allí el filósofo francés sostiene que la característica principal de su trabajo radica en ser una "Historia del Pensamiento", es decir, una historia que define "...las condiciones en las que el ser humano problematiza lo que es, lo que hace y el mundo en el que vive" (Foucault, 2008: 16). El trabajo de una historia del pensamiento consiste, entonces, en determinar las "condiciones" en las que emergen los problemas, esto es, la superficie de elementos que vuelve posibles inquietudes de diferentes órdenes en una sociedad concreta. La pregunta por el estatuto de tales condiciones y por el tipo de relación que éstas mantienen con el pensamiento enmarca los lugares 
en los que Foucault asume el desafío que conlleva moverse en direcciones semejantes a las recorridas por el marxismo y el estructuralismo.

El texto citado sugiere algunos recorridos posibles para precisar la idea de "condiciones", a efectos de especificar la singularidad de la labor de una Historia del Pensamiento en tanto empresa filosófica. En las primeras páginas de la Introducción, Foucault otorga a la noción de "práctica" un lugar preponderante en la constitución histórica de la locura, la delincuencia y la sexualidad. Aquella resulta ser el "medio" en el que se delimitan las experiencias históricas de estos objetos (Foucault, 2008: 10-11). Sin embargo, empleada en singular, la idea de "práctica" no dice mucho ni contribuye a una distinción clara frente al pensamiento de Althusser. En efecto, cuando éste ensaya la reformulación del concepto de "ideología", en la segunda tesis sobre su estructura y funcionamiento, reconoce y ubica la existencia material de la ideología en el orden de las prácticas concretas. Estas no están representadas por cualquier actividad aleatoria, sino que se definen por la pertenencia a uno o varios aparatos ideológicos: “...nosotros hablaremos de actos insertos en prácticas. Y destacaremos que tales prácticas están reguladas por rituales en los cuales se inscriben, en el seno de la existencia material de un aparato ideológico..." (Althusser, 2003: 48). De esta manera, en Althusser la noción de práctica designa un conjunto de actividades, comportamientos, formas de hacer que poseen un principio de regularidad vinculado a un determinado aparato. En otras palabras, las prácticas son tales en función de la regularidad -de las reglas- que ofrece la ideología, a través de los aparatos ideológicos de estado, sobre un conjunto de actos o acciones: “No hay práctica sino por y bajo una ideología" (Althusser, 2003: 51). Esta tesis supone un tipo de relación en la que el principio regulador de las prácticas aparece en cierta medida como extrínseco a las mismas, y esto, fundamentalmente, porque si bien la ideología rige las prácticas desde su propio "interior", remite "exteriormente" a la "lucha de clases", a las formas de producción y a los modos materiales de existencia, como determinantes en última instancia. $^{11}$

\footnotetext{
${ }^{11}$ Cfr., "...las ideologías no "nacen" en los AIE sino que son el producto de las clases sociales tomadas en la lucha de clases: de sus condiciones de existencia, de sus prácticas, de su experiencia de lucha, etcétera" (Althusser, 2003: 66). En el curso del año 1971 Foucault seguía un sendero en cierta forma
} 
La digresión alrededor de la noción de práctica althusseriana permite retornar a Foucault planteando un lugar común al pensamiento de ambos: las prácticas están definidas u organizadas por una regularidad, una serie de reglas que las atraviesan constituyéndolas. Ahora bien, para este último el principio de regularidad no se encuentra en el afuera de las prácticas. Se trate del saber, del gobierno o de la subjetividad, las prácticas no poseen su razón última, su principio de producción, regulación e integración, en un "exterior", sea éste concebido bajo la forma de las relaciones de producción económicas, de la intencionalidad de los individuos o de la ideología de una determinada clase social. De manera concisa, podría decirse que las condiciones de emergencia de problemas, pensamientos y experiencias se definen en un plexo de prácticas o, en términos de Foucault, se organizan en "conjuntos prácticos" (Foucault, 1999: 349), que poseen en sí las relaciones que vuelven efectivo su agrupamiento.

Una Historia del Pensamiento, asumida como una empresa orientada a establecer las condiciones que hacen posibles problemas específicos, pensamientos y experiencias concretas, debe, en consecuencia, dirigir la mirada al nivel de las prácticas, buscando en ellas su principio de inteligibilidad, esto es, su "regularidad", las reglas de producción e integración que las gobiernan y regulan. En el mismo texto, Foucault sitúa los "juegos de verdad" como instancia de producción-integración de prácticas y, por ende, como condición de posibilidad del paso de los acontecimientos a experiencias históricamente definidas:

\footnotetext{
"Una historia que no sería aquella de lo que puede haber de cierto en los conocimientos, sino un análisis de los juegos de verdad, de los juegos de falso y verdadero a partir de los cuales el ser se constituye históricamente como experiencia, es decir, como realidad que puede y debe pensarse a sí misma" (Foucault, 2008: 12).
}

\footnotetext{
semejante al recorrido por Althusser, con la diferencia de que afirmaba que los "acontecimientos discursivos" no son ni del orden del reflejo ni de la expresión de las luchas, sino que constituyen un efecto de las mismas. Foucault se distanciaba tanto de la noción de lucha de clases como de la relación de determinación, así sea en última instancia, entre la clase y el elemento discursivo de la ideología (Foucault, 2012: 216-217).
} 
Se distinguen dos cadencias en el pasaje. Comenzando por el final, el orden del ser adviene a la "realidad" cuando se consolida la trama de un pensamiento que es capaz de pensarlo (que tiene al ser por objeto) a partir de sí mismo (tiene al ser por sujeto). Pensamiento y ser no están escindidos; de aquí la insistencia de Foucault acerca de que el pensamiento no es una mera idealidad, dada la proximidad que tiene con el ser, el cual bajo el estatuto de la "experiencia" hace del pensamiento algo real y de la realidad algo pensable. Por otro lado, el pasaje destaca la centralidad de los "juegos de verdad", es decir, de ese "tamiz" que permite que el ser sea una experiencia "real" -o experiencia del pensar-que se constituye, en forma simultánea, en pensable.

Los juegos de verdad resultan ser el elemento determinante para comprender en qué consiste el objeto sobre el cual dirigir una empresa filosófica en tanto Historia del Pensamiento o Historia de los Sistemas de Pensamiento; en otras palabras, la importancia de los juegos de verdad radica en la posición fundamental que ocupan en la serie "problemas-pensamientos-experiencias". Si las condiciones de posibilidad de estos tres elementos remite a conjuntos prácticos que se definen como tales al remitir a un punto de producción-integración que los recorre, entonces una Historia del Pensamiento es una Historia de la Verdad en tanto convoca bajo su atención los juegos de falso y verdadero que permiten estructurar las prácticas en cuanto problemas, pensamientos, o experiencias. De aquí que la Historia del Pensamiento elaborada por el filósofo francés no coincida con una historia de las representaciones que los hombres poseen sobre algún objeto determinado ni con una historia de las conductas (Foucault, 2008: 17). La actividad de los individuos y sus representaciones no son objeto de la Filosofía, sino de la Historia. ${ }^{12}$

La serie "problemas-pensamientos-experiencias" se organiza alrededor de tres grandes dimensiones o ejes que se correlacionan de diferentes maneras a lo largo de la

\footnotetext{
${ }^{12}$ Carol Bacchi distingue, de manera correcta, dos grandes tradiciones intelectuales en el abordaje y uso de la noción de "problematización". Por un lado, la foucaulteana, que tendría la particularidad de constituirse en tanto producto de las prácticas gubernamentales. $Y$, por otro, la que llama "interpretativista", que se caracterizaría por lo que, justamente, Foucault cuestiona, esto es, el énfasis puesto en la manera en que "la gente", los individuos, los agentes sociales, problematizan lo que hacen (Bacchi, 2015: 3).
} 
historia. Estos tres vectores son "recortes" analíticos que se realizan sobre el fondo de los acontecimientos históricos. En la clase del 5 de enero de 1983 Foucault define, como constitutivos de una experiencia, los campos del saber, las estrategias del poder y las prácticas de subjetivación (Foucault, 2009: 19). Estos tres componentes suponen modos históricos de correlación que los entrelazan a partir de juegos de verdad vinculados a cada uno de ellos. A la luz del nuevo emprendimiento intelectual inaugurado por el volumen segundo de su Historia de la sexualidad, Foucault se refiere de la siguiente manera a sus investigaciones previas, poniendo en el centro de su descripción la noción de "juegos de verdad":

\begin{abstract}
"Tras el estudio de los juegos de verdad unos en relación con otros -tomando el ejemplo de un número determinado de ciencias empíricas en los siglos XVII y XVIII- seguidos por el de los juegos de verdad en relación con las relaciones de poder -con el ejemplo de las prácticas punitivas-, parecía imponerse otro trabajo: estudiarlos en la relación del individuo consigo mismo y en la constitución de sí como sujeto..." (Foucault, 2009: 12).
\end{abstract}

La labor del filósofo se expresa en la puesta en práctica de una Historia del Pensamiento que, paradójicamente, no tiene al pensamiento como su objeto de estudio privilegiado, sino a aquello que lo vuelve posible al trazar su itinerario, demarcar sus límites, establecer objetos, etc. De aquí que la verdad, tomada bajo la forma de "juegos" reglados o "regímenes de veridicción", se convierta en un dominio de análisis fundamental. En otras palabras, la Historia del Pensamiento es una historia de la verdad o, para decirlo más ajustada y kantianamente, una historia de las condiciones histórico-formales que hacen posible la verdad ${ }^{13}$ bajo la modalidad de la vigencia histórica de un pensamiento. En efecto, en la misma obra antes referenciada, Foucault, parafraseándose, afirma que la empresa de una historia de la verdad consiste en: "...analizar, no los comportamientos ni las ideas, no las sociedades ni sus "ideologías", sino las problematizaciones a través de las cuales el ser se da como una

\footnotetext{
${ }^{13} \mathrm{Y}$ esas condiciones que hacen posible la verdad son de dos tipos, prácticas concretas y prácticas discursivas. Las primeras, destacadas fundamentalmente por la genealogía, las segundas por la arqueología. Sin embargo, cuando aquí nos volcamos por el estudio de las "formas" de problematización, apelamos al dominio de las prácticas discursivas y, por ende, traemos a colación la perspectiva analítica correspondiente: la arqueología.
} 
realidad que puede y debe ser pensada por sí misma, y las prácticas a partir de las cuales se forman" (Foucault, 2009: 17). De acuerdo con ello, las formas históricas de problematización, al constituirse en las condiciones de realidad y pensamiento del ser (comportamientos, ideas, etc.), pueden ubicarse en el espacio conceptual ocupado por la idea de juego o régimen de verdad, es decir, en el punto de intersección entre el pensamiento y el "ser/realidad" (eso que ha sido denominado la serie "problemaspensamientos-experiencias"), a lo largo de los ejes del saber, el poder y la subjetividad.

En una dirección, el pensamiento transita circuitos habilitados por formas históricas de problematización; en otra, problematiza las matrices reflexivas consolidadas en una época. Existe, por ende, una correlación entre las nociones de pensamiento, problematización y juegos de verdad que merece explorarse más en el intento de dotar al trabajo filosófico de un objeto propio hacia el cual dirigir el instrumental analítico o, en otras palabras, postular, en la línea abierta por Foucault, que una Historia del Pensamiento consiste, fundamentalmente, en una "analítica de las formas históricas de problematización", tal como se pretende indicar aquí. En efecto, para el francés, el pensamiento no se topa con los objetos que piensa como si estos estuvieran simplemente dispuestos en el mundo, sino que circula a través de objetos que "emergen" y se tornan "recorridos potenciales", "pensamientos pensables". Esto es posible en virtud de la existencia de regímenes de veridicción o juegos de verdad, es decir, por aquella cualidad inherente a las prácticas sociales que permite la constitución de objetos para el pensamiento (Foucault, 1999: 371) al recortar, sobre un fondo indiferenciado de multiplicidades, prácticas de saber, poder y subjetivación. Es esta "trama" (los juegos de verdad ligados a prácticas sociales específicas, y los pensamientos, experiencias y/o problemas que se vuelven posibles -e inciden-sobre los mismos) la que Foucault denomina "problematización":

\footnotetext{
"Problematización no quiere decir representación de un objeto preexistente, así como tampoco creación mediante el discurso de un objeto que no existe. Es el conjunto de prácticas discursivas y no discursivas que hace que algo entre en el juego de lo verdadero y de lo falso y lo constituye como objeto para el pensamiento" (Foucault, 1999: 371).
} 
Este pasaje refuerza la tesis de que el pensamiento ${ }^{14}$ sólo es posible al interior de un circuito diseñado y habilitado por una forma histórica de problematización, esto es, a través de juegos de verdad que operan determinando, por medio de la composición de "objetos", "conceptos" y "posiciones de sujeto", un fondo indiferenciado de acontecimientos múltiples. Ahora bien, si convenimos en decir que una Historia del Pensamiento no es la historia de lo que es representado en el pensamiento, sino de las condiciones -i.e., los juegos de verdad o regímenes de veridicción- que han tornado algo pensable (que han logrado que algo se transforme en objeto para el pensamiento bajo alguna de las tres dimensiones que articulan la experiencia), entonces la referencia a los instrumentos y recursos comprendidos en el tratamiento que Foucault hiciera del "saber", hacia fines de la década de 1960, se torna ineludible.

En otros términos, el orden del saber o de las prácticas discursivas resulta ser el marco general dentro del cual los "juegos de verdad" deben ser analizados como articulando un tipo de problematización específico. Como todo "juego", los juegos de verdad se realizan en base a reglas que permiten definir objetos, establecer posiciones de sujeto y modos de construir conceptos (Foucault, 2013a: 272-273). De esta manera, la Historia del Pensamiento puede ser concebida, genealógicamente, como la “...historia de la emergencia de los juegos de verdad: es la historia de las "veridicciones", entendidas como las formas según las cuales se articulan, en un dominio de cosas, discursos susceptibles de ser llamados verdaderos o falsos" (Foucault, 1999: 364). Pero también una Historia del Pensamiento consiste en el análisis de tales "formas", ${ }^{15}$ los modos en que se diagraman internamente los discursos verdaderos, las reglas de formación que hacen posibles objetos, conceptos y posiciones de sujeto.

\footnotetext{
14 Pero también la experiencia o los problemas, de acuerdo con la estructura de la serie de tres componentes antes mencionada.

${ }^{15}$ Con el uso del término "forma" se busca restituir el sentido que en Kant adquirían los esquemas ordenadores de la sensibilidad y del entendimiento. De acuerdo con ello, las "formas de problematización" podrían pensarse como un a priori histórico, esto es, de modo semejante al estatuto que Foucault otorga a las epistemes en Las palabras y las cosas.
} 
En síntesis, una Historia del Pensamiento definida de esta manera tendría por objeto de estudio los regímenes de veridicción, en tanto cualidad inherente a las prácticas discursivas en el campo del saber, $y$, como estrategia analítica, una "arqueología de las formas históricas de problematización". ${ }^{16}$

\section{3.- La arqueología como estilo de análisis}

Las formas históricas de problematización son la condición de existencia de un campo de experiencias, esto es, de un conjunto de elementos que pueden ser pensados a través de la distinción de tres grandes grupos: objetos de saber, conductas y acciones regladas y prácticas de constitución de subjetividades. Para Foucault, la estrategia analítica de aproximación a estos grupos de elementos, articulados bajo algunos de los términos de la serie "problemas-pensamientos-experiencias", es, como fue mencionado, doble, genealógica y arqueológica. Mientras que el genealogista atiende los azares y las contingencias de la historia en los que las relaciones de fuerza, siempre cambiantes, hacen emerger el acontecimiento del pensar (Foucault, 1997: 49), ${ }^{17}$ la mirada suministrada por la arqueología permite atravesar el nivel de los pensamientos, tomados como "representaciones", "ideas", "ideologías", "problemas", etc., y exponer la operación sostenida de los juegos de verdad que los hacen posibles. En efecto, las formas históricas de problematización no sólo constituyen la trama en la

\footnotetext{
${ }^{16}$ No se descarta aquí el hecho de que una Historia del Pensamiento reúne los dos movimientos analíticos "clásicos" del pensamiento foucaulteano, esto es, una "arqueología de las formas de problematización", proyectada sobre las "formas", en cuanto matrices epistémicas, y una "genealogía de las problematizaciones", dirigida a los procesos en los que determinadas verdades son puestas en crisis.

${ }^{17}$ Un modo clásico en que se ha diferenciado la genealogía de la arqueología consiste en pensar que ésta última atiende el estudio de las prácticas discursivas, y que la primera se centra en las prácticas no discursivas. A pesar de que esta explicación resulta didáctica, compartimos aquí la observación que realiza G. Deleuze, cuando recupera la primacía de lo discursivo por sobre lo no discursivo: "Los enunciados priman sobre las visibilidades" (Deleuze, 2013: 18). Por otro lado, el análisis dinámico de las transformaciones discursivas, empresa que bien puede ser conceptualizada como una actividad genealógica, en cierta medida, se encuentra caracterizado ya en el período en que Foucault objetiva la labor arqueológica. En efecto, en un artículo de 1968, Foucault se refiere al modo en que "arqueológicamente" es posible realizar un análisis de las transformaciones: "Mi problema es: sustituir la forma abstracta, general y monótona del "cambio", a través de la cual, de tan buena gana, se piensa la sucesión, por el análisis de los tipos diferentes de transformación" (Foucault, 2013a: 198).
} 
que se despliegan las reglas de constitución, distribución y entrecruzamiento de los saberes, las relaciones articuladas entre unos individuos y otros, además de aquellas técnicas que tienen el sí mismo como su objeto, sino que están directamente vinculadas con nuestras experiencias y pensamientos efectivos. En relación con esto, Foucault sostiene que una Historia del Pensamiento trata de captar:

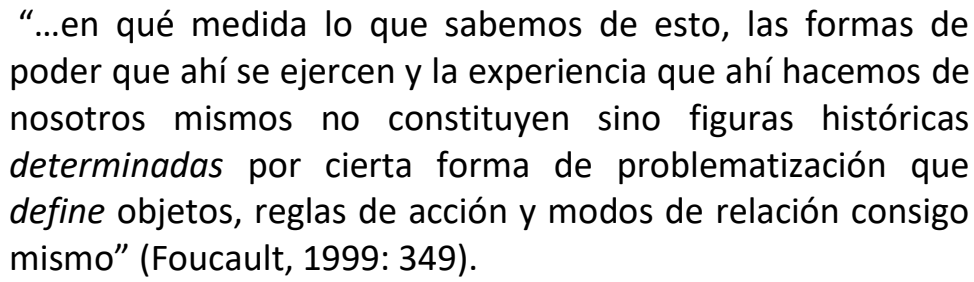

Si el objeto de una Historia del Pensamiento está cifrado por las formas históricas de problematización, el modo de acceso al mismo exige fundamentalmente el trabajo del arqueólogo, aunque el genealogista no deje de ocupar un lugar relevante. Foucault distingue el oficio de las dos grandes perspectivas analíticas acuñadas en su filosofía al sostener que "la dimensión arqueológica del análisis permite analizar las formas mismas de la problematización; su dimensión genealógica, su formación a partir de las prácticas y de sus modificaciones" (Foucault, 2008: 18). Estas expresiones ayudan a cimentar la idea de que la arqueología representa la mirada "cenital", la que cae en vertical sobre las formaciones discursivas de una época y atraviesa sus diferentes capas constitutivas, exponiendo aquellas que hacen posible lo que se muestra en la superficie, las reglas que permiten decir y ver, las condiciones de aparición de lo que es considerado "teoría", "ideología”, "ideas", "problemas", etc. A su lado, la genealogía traza el circuito de las modificaciones históricas, de los devenires y desplazamientos que sufre una determinada forma de problematización en el curso de la historia y en los meandros del proceso de su formación, al tiempo que señala la relación que vincula a la misma con el componente no discursivo de las prácticas, esto es, las disposiciones espaciales, las técnicas y dispositivos de gobierno entre los que se enmarcan.

Las formas históricas de problematización, vistas desde la dimensión arqueológica, remiten directamente a las prácticas discursivas como al espacio sobre el 
cual rastrear el conjunto de relaciones que, bajo la figura del saber, hacen posibles objetos, sujetos y conceptos. En su diccionario, E. Castro sostiene que Foucault amplió el concepto de episteme, utilizado a mediados de los '60 en Las palabras y las cosas, primero a través de la noción de "dispositivo", y luego con la de "práctica"(Castro, 2011: 132). En efecto, en una entrevista realizada en 1977 el filósofo francés reconocía que a través del empleo del término episteme hacía alusión a un tipo de dispositivo específico, caracterizado por el nivel discursivo de las prácticas (Foucault, 1991: 131). Sin embargo, y a la inversa del itinerario resaltado por Castro, en el curso Lecciones sobre la voluntad de saber de 1971, Foucault asumía ya la dimensión de las prácticas en su vertiente discursiva al sostener que las así llamadas "prácticas discursivas" constituyen la superficie a partir de la cual una labor encaminada a reconstruir los "sistemas de pensamiento" se vuelve posible (Foucault, 2012: 247). Ubicadas a cierta distancia de la noción de episteme, y con el interés aún vigente por los "sistemas de pensamiento", la noción de prácticas discursivas remite a un punto de integración caracterizado por el "juego de prescripciones que gobiernan exclusiones y elecciones" (Foucault, 2012: 247), es decir, juegos que, al recortar objetos, establecer el lugar y las determinaciones del sujeto de conocimiento y "fijar las normas para la elaboración de conceptos y teorías" (Foucault, 2012: 247), sugieren la centralidad de los procesos de veridicción en la analítica foucaulteana, tal como serán recorridos en los cursos de años posteriores.

No hay prácticas sin un cierto régimen, esto es, sin la efectividad de un grupo de reglas que bajo su operatoria permite identificar "conjuntos prácticos" con un determinado nivel de especificidad, reconocible y abordable a partir de dos planos diferenciados analíticamente: jurisdicción y veridicción (Foucault, 1982: 66-67). El primero remite a las diferentes formas en que las prácticas prescriben conductas, a través de codificaciones, reglamentos, programas, etc.; mientras que el segundo hace posible abordar un conjunto práctico a partir de los "efectos de verdad" que tienen lugar de acuerdo con la organización y funcionamiento de las reglas de producción de enunciados. El "régimen de veridicción", como determinante en la constitución de un conjunto práctico, permite poner la "producción de lo verdadero y de lo falso en el 
centro del análisis histórico y de la crítica política" (Foucault, 1982: 67), puesto que presenta un correlato con la actualidad a partir de los efectos materiales "reales", inducidos en medio de instituciones y programas de gobierno. ${ }^{18}$

Las prácticas discursivas, en el elemento de sus formas de producción de verdad o veridicción, esto es, desde la perspectiva del régimen o sistema de reglas que gobierna la producción de enunciados, constituyen el fondo sobre el cual se traza la trama que permite, en el trabajo analítico, identificar y describir formas históricas de problematización. En consecuencia, las prácticas discursivas, abordadas desde la relevancia que cobran los juegos de verdad o regímenes de veridicción, demandan para su análisis una mirada que tome en cuenta la constitución de objetos, conceptos, sujetos, etc., y el modo en que dichos elementos condicionan la organización de conjuntos epistémicos, tales como "saberes", "teorías" o "pensamientos". El trabajo filosófico en clave foucaulteana, esto es, la labor de una "historia de los sistemas de pensamiento", ubica y delimita "formas históricas de problematización" al modo de superficies abstractas, pero reales, que hacen posible el surgimiento de tipos específicos de problemas, maneras de pensar y experiencias posibles.

La arqueología foucaulteana, esto es, el punto de vista que se ubica en un nivel determinado a efectos de poner algo en relieve (Foucault, 2013a: 268), puede ser reapropiado y ampliado en los términos de una "analítica de las formas de problematización". La importancia de la articulación arqueológica radica en la actualización de las consecuencias teóricas extraídas de las distancias puestas por Foucault frente a la "historia de las ideas". Parafraseando un conocido pasaje de La arqueología del saber, podríamos decir que una analítica de las formas de problematización: a) no atiende los pensamientos, problemas o representaciones del discurso, si no es en relación con las reglas constitutivas de los mismos; b) no enlaza los problemas y pensamientos de modo tal que entre los mismos exista una sucesión o

\footnotetext{
18 “Estas programaciones de comportamiento, estos regímenes de jurisdicción/veridicción no son unos proyectos de realidad que fracasan. Son unos fragmentos de realidad que inducen unos efectos de los real tan específicos como los de la división de lo verdadero y de lo falso en la manera en cómo los hombres se dirigen, se conducen, a sí mismos y a los demás" (Foucault, 1982: 71). También: "un tipo de racionalidad, una manera de pensar, un programa, una técnica, un conjunto de esfuerzos racionales y coordinados, unos objetivos definidos y continuados, unos instrumentos para alcanzarlos, etc., todo eso es lo real, aunque no pretenda ser la realidad misma ni toda la sociedad" (Foucault, 1982: 46).
} 
continuidad, sino que los piensa en su especificidad discursiva y no-discursiva, en el marco de unas reglas singulares que resultan irreductibles a otras; c) no se interesa ni en la obra ni en el sujeto creador como unidades de análisis, sino en los problemas y pensamientos que atraviesan diferentes obras, textos y documentos en general, ligándolos entre sí; d) finalmente, no trata de extraer de los problemas y pensamientos el fondo secreto de su verdad, sino exponerlos en los modos históricos que asumen su producción, articulación y funcionamiento (Foucault, 2013b: 181-183).

Una analítica de las formas históricas de problematización encuentra en la arqueología, fundamentalmente, la estrategia de acceso a un conjunto de elementos que constituyen un dominio de análisis que puede plantearse en estrecha relación con el objeto propuesto por La arqueología del saber. En efecto, es posible trazar algunas equivalencias generales entre las nociones de "archivo" y "formas de problematización", desde el momento en que Foucault define el archivo como "...la forma en que los acontecimientos discursivos han sido registrados y pueden ser extraídos" (Foucault, 1999: 63); es decir, "...la ley de lo que puede ser dicho, el sistema que rige la aparición de enunciados como acontecimientos singulares..." (Foucault, 2013b: 170). Puesta en estos términos, la noción de formas de problematización se aproximaría a la de archivo, desde el momento en que ambas remiten a la "ley" histórica de lo que puede ser enunciado, problematizado, pensado y, en consecuencia, experimentado.

Esta equivalencia no deja de tener un carácter general. Para extraer alguna potencialidad de la misma, en especial en relación con las prácticas de gobierno (tal como lo expresa la preocupación del filósofo francés hacia fines de la década del setenta), es necesario profundizarla siguiendo el orden de los elementos que se tornan centrales en el pensamiento arqueológico. Pasar de lo general a lo particular en este juego de similitudes implica poner realmente a prueba la afirmación foucaulteana acerca de la posibilidad de analizar la "racionalidad política" bajo los mismos términos que hacen posible un análisis de las ciencias (Foucault, 2013c: 255). En efecto, para elevar al máximo las potencialidades de un recorte de la noción de problematización realizado en los términos de una arqueología del saber es preciso atender las ideas de 
"juegos de verdad" o "régimen de veridicción". Estas representan el núcleo -muchas veces olvidado- de la noción de problematización, y habilitan su reconstrucción en términos arqueológicos con mayor legitimidad que las aproximaciones realizadas desde el punto de vista de una ética del pensamiento.

Si los juegos de verdad o regímenes de veridicción aparecen definidos como aquellas prácticas que hacen entrar algo en el orden de las distinciones entre lo verdadero y lo falso y, por lo tanto, las proyectan a la existencia como objetos para el pensamiento y la acción, entonces sus particularidades pueden ser exploradas adoptando los elementos presentes en el análisis del "saber", desarrollado en La arqueología del saber. Como fue mencionado, el archivo y las formas históricas de problematización se asemejan en tanto y en cuanto el primero es a la arqueología lo que las segundas son para una Historia de los sistemas de pensamiento. En medio de este juego de equivalencias y similitudes, la noción de archivo nos permite comprender la de forma de problematización, en tanto objeto de investigación. En su diccionario, Castro precisa la noción de archivo al referirse al mismo como "...el sistema de las condiciones históricas de posibilidad de los enunciados" (Castro, 2011: 38). Esta mirada aproxima al archivo a la noción de "forma" al considerarse ésta como el a priori histórico que establece las condiciones de posibilidad del pensamiento. En resumidas cuentas, asumiremos aquí que la diferencia entre la noción de archivo y la de forma no reside tanto en un nivel conceptual como contextual, es decir, relativo al tipo de trabajo que Foucault se encuentra desarrollando al momento en que emplea una u otra. Siendo que el objetivo de su empresa intelectual hacia fines de los '60 no difiere en gran medida de la emprendida a comienzos de los ${ }^{\prime} 80,{ }^{19}$ la noción de forma de problematización puede ser pensada bajo las características asignadas al archivo y asumida como una ampliación de este último concepto.

La naturaleza apriorística e histórica del archivo/forma, ${ }^{20}$ exige atender el modo en que hace posible tanto el enunciado como el pensamiento. Entre ambos grupos de

\footnotetext{
${ }^{19}$ Esto es así si convenimos en definir el trabajo filosófico de Foucault como una Historia de los sistemas de pensamiento.

${ }^{20}$ Hablamos aquí de un "apriorismo histórico" en el mismo sentido en que Foucault piensa las epistemes en Las palabras y las cosas: "Es evidente que tal análisis no incumbe a la historia de las ideas o de las ciencias: es más bien un estudio que se esfuerza por reencontrar aquello a partir de lo cual han sido
} 
elementos, esto es, archivo/forma, por un lado, y enunciado/pensamiento, por otro, se extiende la idea de "regularidad" como aquello que los vincula. En sentido estricto, lo que define el apriorismo del archivo/forma es una cierta regularidad en la constitución de elementos del pensamiento, es decir, en la producción de objetos, conceptos, posiciones de sujeto y estrategias. Es la presencia de tal regularidad en la formación de los componentes mínimos del pensamiento lo que ilumina el perímetro de una forma histórica, y permite pensarla bajo la noción de archivo. En La arqueología del saber, Foucault afirma que la idea de "regularidad" alude, “...para toda actuación verbal cualquiera que sea (extraordinaria o trivial, única en su género o mil veces repetida) el conjunto de las condiciones en que se ejerce la función enunciativa que asegura y define su existencia" Foucault, 2013b: 188). La regularidad es el sustrato material de los enunciados $\mathrm{y}$, por ende, de las frases y proposiciones. Como tal no mienta un promedio, no alude a una suerte de término medio común a un conjunto de manifestaciones verbales; de hecho es posible hablar de regularidad ante un único acontecimiento enunciativo. Foucault dice que la regularidad "...no caracteriza una posición central determinada entre los límites de una curva estadística -no puede, pues, valer como indicio de frecuencia o probabilidad-; especifica un campo efectivo de aparición" (Foucault, 2013b: 188). Dicho campo efectivo de aparición es reconocido como archivo (o "forma de problematización", de acuerdo con la equivalencia ensayada), puesto que es el mismo que reúne el "conjunto de las condiciones" bajo las cuales es posible enunciar y pensar.

No hay enunciado ni pensamiento sin la remisión a una regla o a un conjunto de reglas de formación y existencia. He aquí lo regular en el discurso (Foucault, 2013b: 141-151). ${ }^{21}$ Foucault no adopta la noción de regularidad como manera de designar un denominador común que atravesaría las prácticas discursivas (Foucault, 2012: 247-

posibles conocimientos y teorías; según cuál espacio de orden el saber se ha constituido; sobre el fondo de qué a priori histórico y en qué elemento de positividad han podido aparecer las ideas, constituirse las ciencias, reflexionarse las experiencias en las filosofías, formarse las racionalidades para anularse y desvanecerse quizá pronto" (Foucault, 2008b: 15).

${ }^{21}$ Por "discurso" Foucault refiere a un conjunto de enunciados agrupados bajo un mismo sistema de formación (discursiva), esto es, atravesados por un mismo régimen general. 
248), ${ }^{22}$ sino como remisión directa a reglas constitutivas. Dichas reglas son el a priori de la enunciación y, por ende, del pensamiento. En efecto, "todo enunciado es portador de cierta regularidad, y no puede ser disociado de ella" (Foucault, 2013b: 188). Sin embargo, pese a su formulación apriorística, las reglas no están "antes" del enunciado, ni lógica ni temporalmente, sino que las reglas son el propio enunciado. Esto es así en tanto y en cuanto la naturaleza del enunciado no es la de la frase o proposición, por más que puede ser expresado por una u otra, sino que, antes bien, el enunciado es una función que tiene lugar en lo dicho o, mejor, que hace posible lo dicho (y lo pensable).

Como función, el enunciado es una regla, pues vincula dos conjuntos de variables bajo una modalidad determinada, pero también es una máquina, dado que a un cierto input le hace corresponder un output singular. En otras palabras, para Foucault el enunciado no es un objeto comparable a la frase o a la proposición, no es individualizable como lo pueden ser algunos de estos dos elementos, "...se trata más bien de una función que se ejerce verticalmente con relación a esas diversas unidades, y que permite decir, a propósito de una serie de signos, si están presentes en ella o no" (Foucault, 2013b: 115). De aquí que la función enunciativa sea la de reunir, agrupar, posicionar, posibilitar el sentido de signos que serán, consecuentemente, frases o proposiciones. En otras palabras, el enunciado hace existir lo dicho; representa, como fue destacado anteriormente, su condición de posibilidad. ${ }^{23}$

El trabajo arqueológico se dirige claramente al espacio que es posible trazar entre el enunciado, o los enunciados, y la "formación discursiva", en tanto y en cuanto representa el “...principio de dispersión y de repartición de los enunciados” (Foucault, 2013b: 141). Esto supone asumir que los enunciados constituyen redes enunciativas identificables alrededor de una cierta trama que los reúne. Foucault dirá que:

\footnotetext{
${ }^{22}$ Las regularidades de una práctica discursiva se manifiestan a través de obras particulares, sin coincidir con las mismas. También se expresan a través de ciertas disciplinas, aunque, nuevamente, sus límites pueden no coinciden enteramente con ellas.

${ }^{23}$ El enunciado "...no es en sí mismo una unidad, sino una función que cruza un dominio de estructuras y de unidades posibles y que las hace aparecer, con contenidos concretos, en el tiempo y en el espacio" (Foucault, 2013b: 115).
} 
"La regularidad de los enunciados está definida por la misma formación discursiva. La formación discursiva se caracteriza por una dispersión de hecho, ya que es para los enunciados, no una condición de posibilidad, sino una ley de coexistencia..." (Foucault, 2013b: 153).

Presentada como aquello que reúne -la ley de coexistencia de los enunciados-, la noción de formación discursiva permite abrir camino hacia el estudio de sistemas de formación revestidos del estatuto de saberes, ciencias, disciplinas, etc., puesto que establece todo un orden de regularidades actualizado permanentemente en la puesta en existencia de objetos, conceptos, posiciones de sujetos y estrategias temáticas. ${ }^{24}$ En resumidas cuentas, la formación discursiva establece el régimen de correlación de los enunciados $y$, en consecuencia, la ley que hace posible la existencia de frases $y$ proposiciones.

Los enunciados tienen una relación de pertenencia con las formaciones discursivas, puesto que las mismas permiten identificar y analizar algunos de los elementos epistemológicos más clásicos en el campo del saber, como objetos, conceptos y sujetos. La arqueología atiende la correlación de los enunciados en el elemento de las formaciones discursivas incorporando un componente más, un nivel más al ejercicio de la analítica: el plano del archivo. Éste representa el "sistema general de la formación y la transformación de los enunciados" (Foucault, 2013b: 171). Si la formación discursiva es lo que permite ubicar en un mismo espacio acontecimientos enunciativos, el archivo es lo que refleja el sistema conjunto de su funcionamiento y enunciabilidad. En otras palabras, la formación discursiva establece el régimen a través del cual se rige el agrupamiento o dispersión de los enunciados, así como todas las operaciones vinculadas con la función enunciativa, esto es, la puesta en existencia de los elementos epistemológicos fundamentales en la estructuración del saber (objetos, conceptos, sujetos, etc.). De forma concomitante, el archivo es el sistema de

\footnotetext{
24 "En el caso de que se pudiera describir, entre cierto número de enunciados, semejante sistema de dispersión, en el caso de que entre los objetos, los tipos de enunciación, los conceptos, las elecciones temáticas, se pudiera definir una regularidad (un orden, correlaciones, posiciones en funcionamientos, transformaciones), se dirá, por convención, que se trata de una formación discursiva..." (Foucault, 2013b: 55).
} 
correlaciones entre enunciados (Foucault, 2013b: 169) que evidencia una formación discursiva al ser analizada desde el punto de vista arqueológico (Foucault, 2013b: 173).

Si el enunciado mienta una función, entonces la formación discursiva invoca un régimen y el archivo designa el nivel de la prácticas reguladas en tanto a priori histórico, esto es, como condición de posibilidad de las formas que el saber adquiere en una época determinada. Dependiendo del nivel en el que se sitúe la mirada del arqueólogo, los enunciados, o sus sistemas de dispersión y correlación, adoptarán una mayor centralidad en la investigación. Sin embargo, se trate de uno u otro, lo cierto es que el enunciado se instituye en el centro de interés del trabajo foucaulteano de mediados de los años 60 . Auscultado por el lado de sus determinaciones, el enunciado nos conduce a los sistemas de formación, a las prácticas regulares que los reúnen y los esparcen conformando campos enunciativos teórico-prácticos, pasibles de ser reconocidos bajo las figuras de las ciencias, los reglamentos, las leyes, los programas políticos, las instituciones, etc. (Foucault, 2013a: 254). Mientras que, en dirección a lo que arroja a la existencia, el enunciado debe explorarse en relación con lo que Deleuze llama sus "derivados" o "funciones derivadas", esto es: objetos, conceptos, sujetos y estrategias (Deleuze, 2013: 126-132). En efecto, y de acuerdo con Foucault, “...el menor enunciado... desencadena todo el juego de las reglas según las cuales están formados su objeto, su modalidad, los conceptos que utiliza y la estrategia de que forma parte" (Foucault, 2013b: 191).

Una aproximación a las formas de problematización desde el andamiaje conceptual propio de la arqueología invita a pensar en el modo en que los problemas de una época se vuelven posibles sobre la base de una determinada disposición de los elementos fundamentales para el pensamiento. Es así que si por "forma" la atención se detiene fundamentalmente en el plano del archivo y de la formación discursiva, explicitando el juego de regularidades que caracteriza la ordenación y correlación de enunciados, la "problematización" mienta más bien aquello que tiene lugar en el plano del pensamiento, al componer un espacio discursivo construido a partir de objetos, modalidades de enunciación o posiciones de sujeto, conceptos y estrategias enunciativas. 
Tomado como "máquina" se realza el estatuto de función que el enunciado posee, lo que implica que los elementos derivados o generados por él dependen, en el orden de lo que los mueve a existir, de la vigencia o alteración de otros enunciados y del campo enunciativo. De esta manera, cada uno de los componentes fundamentales del pensamiento debe exponer, en su determinación, el vínculo que lo hace existir como tal a partir de los enunciados. Desde esta perspectiva, el "objeto", como derivado de la función enunciativa, no hace referencia a nada que se pueda encontrar en el mundo, en el más allá del discurso, en los "hechos de la realidad", sino más bien a una superficie que será ocupada, a una posición que será tomada, a un espacio de emergencia que dará lugar al surgimiento y a la distinción de una cosa frente a otras (Foucault, 2013b: 120). Foucault expresa el interés arqueológico en los "objetos" del discurso al afirmar que la labor intelectual consiste en:

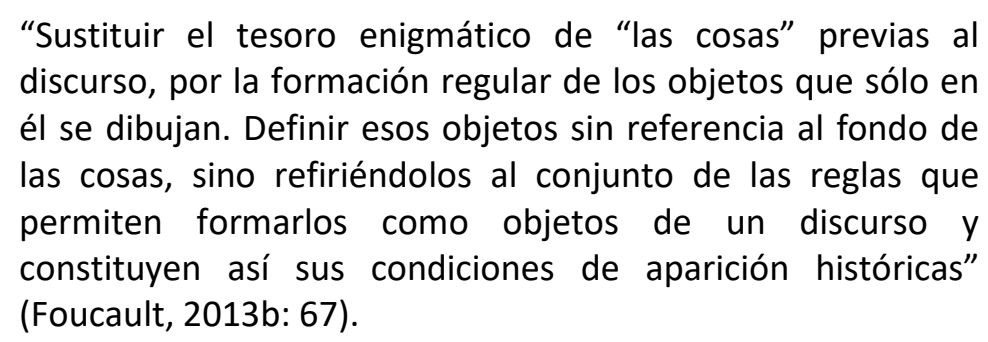

Para el filósofo francés los objetos se derivan de la función referencial que tienen el enunciado al establecer un campo regular de posibilidades. El objeto es lo que puede aparecer entre un número finito de posibilidades, en una superficie determinada y en relación específica con otros elementos. No hay objeto sin función referencial, no hay objeto sin una regla de formación que lo proyecte a la existencia. Así conceptualizado, el objeto es un derivado de las prácticas discursivas, no de las "cosas", sin embargo, su dependencia de las prácticas no lo transforma en un duplicado de las condiciones institucionales, económicas y políticas en medio de las cuales éstas tienen lugar.

El otro elemento derivado de la función enunciativa y que da lugar al pensamiento y a los problemas que lo tipifican en una época es el "sujeto". Como tal, Foucault se refiere al equivalente del "sujeto de la enunciación" en una frase, o de la "modalidad enunciativa". De manera semejante al objeto, como función derivada del 
enunciado, el sujeto cumple las mismas condiciones. No remite a un individuo determinado, de carne y hueso, o a una conciencia. No es el lugar sintético que condensa en sí las diversas expresiones y acciones que se suscitan en el discurso. Es un derivado de un sistema de reglas que lo constituye como tal asignándole atribuciones. De acuerdo con ello, sujeto es aquel a quién corresponde un determinado "estatuto" en función del cual adquieren una cierta veracidad y hasta poder instituyente (Foucault, 2013b: 70). Es por ello que la modalidad enunciativa o posición de sujeto remite, necesariamente, a instituciones y a prácticas específicas, mediadas por reglamentos (Foucault, 2013b: 72-73). Sin embargo, en una determinada formación discursiva, las modalidades enunciativas normalmente reenvían hacia varios sujetos, puesto que se entrecruzan diversos estatutos, ámbitos, y prácticas normalizadas. De aquí que la noción de sujeto, para Foucault, refiera no a un punto de síntesis o unificación, sino a una "dispersión", 25 a una red de atribuciones y facultades.

El siguiente elemento, central para el pensamiento, es el concepto. El trabajo arqueológico no toma a los conceptos como relevantes desde el punto de vista de su enumeración, de su clasificación, de su puesta en comparación, etc. Los conceptos son importantes para la arqueología porque remiten a reglas de formación y de dispersión que los agrupan en esquemas. El concepto es una línea en una figura, es un trazo en un esquema. Lo fundamental aquí es que no hay concepto sin esquema y que éste último alude a un régimen enunciativo que lo traza una y otra vez, en sus similitudes y variaciones. Foucault destaca que: "estos esquemas permiten describir, no las leyes de construcción interna de los conceptos, no su génesis progresiva e individual en el espíritu de un hombre, sino su dispersión anónima a través de textos, libros y obras" (Foucault, 2013b: 81). Los conceptos no dejan de remitir a un campo pre-conceptual, que Foucault comprende como el espacio signado por las reglas de formación conceptual. Dichas reglas de formación no son del orden de la idealidad ni de la empiricidad, sino de la materialidad del discurso que se articula en el nivel mismo de las funciones enunciativas.

\footnotetext{
25 “En el análisis propuesto, las diversas modalidades de enunciación, en lugar de remitir a la síntesis o a la función unificadora de un sujeto, manifiestan su dispersión. A los diversos estatutos, a los diversos ámbitos, a las diversas posiciones que puede ocupar o recibir cuando pronuncia un discurso. A la discontinuidad de los planos desde los que habla" (Foucault, 2013b: 75).
} 
La última noción que la arqueología sugiere como modo de articulación del saber en su abordaje analítico es la de "estrategias" o "estrategias enunciativas". Bajo esta designación Foucault comprende la noción clásica de "teorías", sin embargo pregunta por el modo en que las mismas se despliegan al interior de una formación discursiva determinada. Así, la existencia de temas y teorías responde a estrategias, cuyo nivel de formación se encuentra, nuevamente, posibilitado por las funciones enunciativas en el nivel del plexo de enunciados que compone la formación de la cual se trate. Las estrategias teóricas no son producto de la decisión de un sujeto, del interés de un colectivo, o de cualquier otra instancia cuyo obrar se mueva por una finalidad consciente (proyecto, opiniones); las determinaciones de las elecciones teóricas son el resultado de la interacción entre un conjunto de variables, que pueden estar vinculadas a lo que Foucault denomina "prácticas no discursivas" (Foucault, 2013b: 91), y un orden de posibilidades establecido dentro de una formación discursiva. En efecto, las formaciones discursivas, en tanto plexo de enunciados interrelacionados y reglas de formación de objetos, posiciones de sujeto y conceptos, ofrecen operaciones combinatorias finitas que se efectivizan históricamente sin coincidir con la totalidad de las que podrían efectuarse. Esta diferencia entre el orden de posibilidades de elecciones teóricas, organizado a partir de una determinada formación, y las elecciones efectivamente llevadas a cabo constituye el foco de interés de la arqueología foucaulteana. ${ }^{26}$ Una pista que, de acuerdo con el filósofo francés, permite describir las determinaciones y recortes teóricos obrados en una formación discursiva remite a la "economía de la constelación discursiva" (Foucault, 2013b: 89). Con ello Foucault refiere al juego de aproximaciones y distancias (analogía, oposición, complementariedad, etc.) que se desarrolla entre diferentes discursos en una época determinada. Según el autor, es éste juego una de las variables que determina las elecciones teóricas:

\footnotetext{
"Todo este juego de relaciones constituye un principio de determinación que permite o excluye en el interior de un
}

\footnotetext{
26 "Para dar cuenta de las elecciones que se han realizado entre todas aquellas que hubieran podido realizarse (y éstas únicamente) es preciso describir instancias específicas de decisión" (Foucault, 2013b: 89).
} 
discurso dado cierto número de enunciados: hay sistematizaciones conceptuales, encadenamientos enunciativos, grupos y organizaciones de objetos que hubieran sido posibles (y cuya ausencia en el plano de sus reglas propias de formación nada puede justificar), pero que han sido excluidos por una constelación discursiva de un nivel más elevado y de una extensión mayor" (Foucault, 2013b: 90).

Es clara la deuda que este pasaje tiene con respecto al trabajo desarrollado en Las palabras y las cosas. Foucault parece recuperar el movimiento que ponía en relación un conjunto de saberes entre sí, bajo la noción amplia de episteme, y otorgar, al juego de sus interferencias, la posibilidad del recorte de las estrategias teóricas. Sea una cosa o la otra, lo cierto es que para el año 1969 Foucault reconocía que sobre este asunto no dejaba de andar a tientas (Foucault, 2013b: 88).

Más allá de la honestidad intelectual, expresada en el reconocimiento de los límites de su investigación, Foucault detectó dos características de las elecciones teóricas que resultan relevantes. La primera se refiere al hecho de que dos, o más, elecciones teóricas opuestas pueden pertenecer a una misma formación discursiva; la segunda, es la explicitación del problema del tipo de relación que las elecciones teóricas mantienen con el plano de lo no discursivo (Foucault, 2013b: 92). Ambas particularidades aproximan la temática de las formaciones enunciativas y del análisis arqueológico de las reglas de formación al campo político, dado que sugieren tempranamente los anudamientos que luego Foucault explorará entre prácticas de gobierno y juegos de verdad. ${ }^{27}$

\section{Conclusión}

La mayor parte de las discusiones metodológicas alrededor de la obra de Michel Foucault ha tendido a poner de relieve el "momento nietzscheano" de su pensamiento. La genealogía fue concebida entonces como una estrategia eficaz frente a un saber histórico lastrado por la centralidad de los universales, la permanencia de

\footnotetext{
27 “...las reglas de formación tienen su lugar no en la "mentalidad” o la conciencia de los individuos, sino en el discurso mismo; se imponen, por consiguiente, según una especie de anonimato uniforme, a todos los individuos que se disponen a hablar en ese campo discursivo" (Foucault, 2013b: 84).
} 
los procesos, las invariantes, las finalidades, etc. De aquí que el programa de trabajo foucaulteano haya sido concebido en muchas ocasiones como formando parte de la tradición de una "escuela crítica", organizada sobre la denuncia de la apariencia de lo inmutable y ofreciendo como contrapartida la valoración de una ontología de la variancia, el cambio, la mutación, el azar de los procesos, etc.

Un poco a contracorriente de tales recuperaciones, este escrito pretendió volver sobre el "momento kantiano" del pensador francés. El mismo estaría caracterizado por la atención puesta en el a priori histórico, es decir, en las condiciones discursivas que hacen posible la emergencia de enunciados verdaderos en una época determinada. Vista desde esta perspectiva, la cuestión de la metodología en Foucault puede organizarse a partir del problema de la existencia de aquello que no existe, lo cual conduce necesariamente a la cuestión de la verdad, a su emergencia como tal, su permanencia o regularidad, antes que a su estatuto evanescente o ficcional.

Para afianzar esta tesitura, el artículo ha ensayado el abordaje conjunto de tres elementos caros al programa de trabajo foucaulteano: una historia del pensamiento, comprendida como empresa de trabajo intelectual; las formas de problematización, en tanto objetos de conocimiento sobre los cuales dicha empresa se pliega; $y$, finalmente, la arqueología como estilo de trabajo, perspectiva o método, por medio de la cual se accedería a las formas de problematización. A los efectos de darle consistencia a esta propuesta, se indagó, en una primera instancia, sobre el doble sentido del término "problematización" en la obra foucaulteana. Se realzó, específicamente, su carácter de objeto de conocimiento frente al de actividad intelectual o cualidad del trabajo crítico. La diferencia entre un sentido y otro se marcó a partir de las contribuciones de C. Bacchi, haciendo hincapié en la centralidad que la politóloga le otorga, en su lectura, a la noción de "proceso". Para esta autora, ambos sentidos, remiten a las variaciones y transformaciones histórico-sociales que producen objetos para el pensamiento, con lo cual la problematización aparece siempre como índice del cambio o de la desustancialización. Desde esta perspectiva, Bacchi privilegia, como modos de acceso a la problematización, la genealogía y la "eventualización", puesto que ambas estrategias permiten desnaturalizar y reubicar en el contexto de la historicidad los 
grandes problemas alrededor de los que se organizan los programas de gobierno e intervención pública.

El rodeo a través de la concepción de Bacchi permitió tornar evidente la necesidad de reparar antes que en los procesos, en las formas discursivas que estos adquieren cuando se consolidan históricamente. Para ello se han seguido los pasos del proyecto foucaulteano de una historia de los sistemas de pensamiento, en el que el centro de la escena es ocupado por la relevancia de los juegos de verdad, en tanto principios organizadores e integradores de las prácticas de saber, poder y subjetivación. Pero una historia del pensamiento que se pliega sobre los juegos de verdad exige una analítica de las formas antes que de los procesos. En este punto se abren los caminos que separan, aunque no excluyen, genealogía y arqueología como modos de acceso a la problematización. La primera -genealogía- sugiere orientarse sobre los procesos que constituyen los juegos de verdad, mientras que la segunda arqueología- exige determinar las formas que dan lugar al pensamiento, a partir de la organización de elementos discursivos que articulan problemas y soluciones.

La última estación del escrito está caracterizada por una recuperación de las nociones fundamentales de La arqueología del saber y un ejercicio de equivalencias y analogías posibles con las formas de problematización y el pensamiento. De acuerdo con ello, es posible considerar a las formas de problematización como un a priori histórico, al modo en que el Foucault de fines de los '60 comprende el archivo, para reflexionar sobre los regímenes de dispersión de las formaciones discursivas y las funciones enunciativas que dan forma a los problemas y al pensamiento. De esta manera, quizá, la empresa intelectual de una historia de los sistemas de pensamiento pueda recortarse metodológica y epistemológicamente sobre las formas de problematización y los juegos de verdad que le son inherentes, en tanto objetos de inquietud, y la arqueología como modo de acceso privilegiado a los mismos.

De tener la tesitura aquí planteada algún grado de verosimilitud, el pensamiento de Foucault podría ser abordado -en lo que respecta a su uso para nuevas estrategias de análisis- haciendo prevalecer aquellas instancias que parecen desentonar con la referida impronta nietzscheana. Una recuperación realizada bajo 
esta clave opondría al cambio, la evanescencia y la mutación la permanencia, la regularidad y la estabilidad de las formas de problematización. En la misma dirección, una historia de los sistemas de pensamiento debería asumirse como un modo de relacionarse con el presente, con lo actual, antes que con el pasado. En otras palabras, cierto privilegio de lo regular y actual frente al cambio y la historia; Kant antes que Nietzsche. No se trata, claro está, de opciones excluyentes, sino de cierta "prudencia metodológica" (al decir de Foucault), orientada a evitar la seducción de un historicismo crítico incapaz de avanzar más allá de la denuncia de los universales.

\section{Bibliografía}

Althusser, L. (2003). Ideología y aparatos ideológicos de Estado, Bs. As.: Nueva Visión.

Bacchi, C. (2010). "Foucault, Policy and Rule: Challenging the Problem-Solving Paradigm", FREIA's tekstserie, Aalborg, Institut for Historie, Internationale Studier og Samfundsforhold, Aalborg Universitet, No. 74.

Bacchi, C. (2012). "Why Study Problematizations? Making Politics Visible", en Open Journal of Political Science, Vol. 2, No. 1.

-. (2015). "The Turn to Problematization: Political Implications of Contrasting Interpretative and Poststructural Adaptations", en Open Journal of Political Science.

Barnett, C. (2015). "On Problematization. Elaborations on a Theme in "Late Foucault"”, en Non Site, URL: http://nonsite.org/article/on-problematization\#foot src 29$\underline{8843}$.

Brossat, A. y Chevallier, P. (2016). "The Materiality of a Working Life. An interview whit Daniel Defert", en Foucault Studies, translate by Colin Gordon, № 21.

Castro, E. (2011). Diccionario Foucault. Temas, conceptos y autores, Bs. As.: Siglo XXI.

Deacon, R. (2000). "Theory as Practice: Foucault's Concept of Problematization", en Telos, 118.

Deleuze, G. (2013). "El saber: curso sobre Foucault", Bs. As.: Cactus.

Díaz Marsá, M. (2008). “¿Qué quiere decir pensar? Acerca de la noción de problematización en Michel Foucault", en Daímon. Revista de Filosofía, N 43.

Foucault, M. (1982). La imposible prisión, Barcelona: Anagrama.

. (1991). Saber y Verdad, Madrid: La Piqueta.

(1997), Nietzsche, la genealogía, la historia, Valencia: Pre-Textos.

(1999). Estética, ética y hermenéutica. Obras esenciales, volumen III, Barcelona: Paidós.

. (2008). Historia de la sexualidad 2. El uso de los placeres, Bs. As.: Siglo XXI. (2008b). Las palabras y las cosas, trad. Elsa Frost, Bs. As.: Siglo XXI Editores Argentina.

(2009). El gobierno de sí y de los otros: curso en el Collège de France (19821983), Bs. As.: FCE. 
(2012). Lecciones sobre la voluntad de saber, Bs. As.: FCE.

. (2013a). ¿Qué es usted profesor Foucault?, Bs. As.: Siglo XXI.

(2013b). La arqueología del saber, Bs. As.: Siglo XXI.

(2013c). La inquietud por la verdad: escritos sobre la sexualidad y el sujeto, Bs. As.: Siglo XXI Editores.

(2015). La ética del pensamiento. Para una crítica de lo que somos, Madrid: Biblioteca Nueva.

--------. (2017). Discurso y verdad. Conferencias sobre el coraje de decirlo todo, Bs. As.: Siglo XXI.

Gordon, C. (2016). "The Cambridge Foucault Lexicon (review)", en History of the Human Sciences, vol. 29.

Potte-Bonneville, M. (2007). Michel Foucault, la inquietud de la historia, Bs. As.: Manantial.

Restrepo, E. (2008). “Cuestiones de método: eventualización y problematización en Foucault", en Tabula Rasa, Bogotá, Colombia, $N^{\circ} 8$.

Vega, G. (2012). "Aufklärung y parrhesía en Michel Foucault", en Logos, Bogotá, Colombia: Universidad de La Salle, № 22. 